European Journal of Economic and Social Systems

Vol. 15, N³ (2001) 109-129

\title{
EVOLUTIONARY DYNAMICS OF THE TRANSITIONS ACROSS THE NASH EQUILIBRIA OF A TACIT COORDINATION GAME
}

\author{
JASMINA ARIFOVIC ${ }^{1}$
}

\begin{abstract}
We investigate evolutionary adaptation in a repeated coordination game with strategic uncertainty. The game is characterized by multiplicity of stationary and cyclical Nash equilibria. Monomorphic equilibria of the game are neutrally stable. The results of simulations in which players use the genetic algorithm to update their strategies show that, regardless of the number of players that participate in the game, any equilibrium can be reached. However, the time spent in high effort equilibria is negatively related to the number of players. Finally, regardless of the group size, players play best response actions most of the time. The dynamics of our model capture the main features of the behavior observed in the experiments with human subjects. As the evolutionary dynamics generate persistent fluctuations in the level of effort, these results are also relevant for macroeconomic models with strategic complementarities where fluctuations in economic activity can occur as a result of shifts in agents' expectations.
\end{abstract}

Classification Codes. D0, E32, E39, Z00.

\section{INTRODUCTION}

Coordination games with strategic uncertainty are usually analyzed in the context of a team-production model in which players make decisions about the level of effort they want to contribute towards production of a joint final good. When making their decisions, players do not have information on the actions taken by their fellow participants. The amount of final good produced is determined by the minimum amount of effort. Players obtain the highest payoff when each player

Keywords and phrases: Coordination games, evolutionary dynamics, transition across equilibria.

1 Simon Fraser University. e-mail: arifovic@sfu.ca

(C) EDP Sciences 2002 
contributes the maximum feasible amount of effort, but there is an element of strategic uncertainty since players are not sure about what the others will do. Thus, these games are characterized by multiplicity of equilibria that can be Pareto ranked.

Evidence from the experiments with human subjects suggests that neither the payoff dominance nor risk dominance equilibrium selection criteria can be used as an equilibrium selection device. Experimental evidence also shows that group size, payoff parameter values, (Van Huyck et al. 1990) and the number of repetitions of the game (Berninghaus and Ehrhart 1998) have impact on the equilibrium selection process. In the context of a repeated stage game, different models of learning and adaptation capture the behavior observed in the large group experimental treatment. However, they cannot account for the behavior observed in the small group treatment. In addition, they do not address the impact of the number of repetitions on the equilibrium selection in large group treatments.

We depart from the previous studies and formulate our model as a repeated game. The repeated character of the game increases the number of Nash equilibria, both stationary and cyclical. Most of the equilibria of the repeated game are weak, i.e. equilibrium strategies are not unique best responses. We analyze evolutionary stability of these equilibria in the context of a game with monomorphic population, infinite number of players and pairwise matching, and find that most of these equilibria are neutrally stable. This implies that, under certain conditions, invading strategies will not necessarily be driven out of a population. Because these equilibria are neutrally stable, transition from one Nash equilibrium to the other is made possible through the effects of mutation.

The results obtained with monomorphic populations are suggestive of the dynamics that take place in the evolution of polymorphic populations with finite number of players. We study the evolution of such polymorphic populations with finite number of players using the genetic algorithm ${ }^{2}$.

The results of our simulations show that evolutionary dynamics take populations of strategies through different Nash equilibria regardless of the group size. They also show that populations of all group sizes spend significant fractions of time close to one of the Nash equilibria of the game. However, the fraction of time spent close to the high-effort equilibria decreases monotonically with increases in the number of players in a group. Small group sizes spend more time in high effort equilibria, while large group sizes spend more time in the low effort equilibria.

\footnotetext{
${ }^{2}$ Evolutionary algorithms such as genetic algorithms, genetic programming, evolutionary programming etc. have several advantages in modeling of adaptation of economic agents. They impose low requirement on the computational ability of economic agents. They allow for modeling the heterogeneity of agents' beliefs. Survival of decision rules depends on their performance, measured by the payoff that agents receive by employing them. Also, these algorithms perform better than models with rational agents or alternative models of adaptive behavior in terms of their ability to explain the features observed in experimental economies (see, for example, Arifovic 1994, 1996a).
} 
It is worthwhile to point out the significance of these results for modeling of fluctuations in macroeconomic activity that do not result from changes in fundamentals but rather from fluctuations in agents' expectations. The team production game studied in this paper can be interpreted as a version of a model with strategic complementarities (e.g. Bryant 1983 and Cooper and John 1988) studied in macroeconomic theory ${ }^{3}$. These macroeconomic models are also characterized by multiplicity of equilibria that reflect different levels of economic activity. The movement from equilibria with high to equilibria with the low level of economic activity can occur because of the changes in agents' expectations. The results presented in the paper provide a description of the way in which these changes and transitions can be initiated and realized. Evolutionary dynamics take the economies through different stationary and cyclical equilibria resulting in persistent fluctuations of the level of economic activity.

Section 2 of the paper describes the basic stage game, the results obtained in the experiments with human subjects and the results of application of other adaptive models. Description of the repeated game and its Nash equilibria is given in Section 3. This section also contains the analysis of evolutionary stability of the monomorphic equilibria Genetic algorithm application is described in Section 4. Results of simulations are presented in Section 5. Discussion of the relevance of the results for macroeconomic modeling is provided in Section 6. Finally, Section 7 contains concluding remarks.

\section{Minimum EFFort Game}

First, we describe a version of the minimum effort game that was used in Van Huyck et al. (1990) experimental design. It is a team-production game where a player $i, i \in[1, N]$ makes a decision about the level of effort $e_{i}$ that she contributes to the joint production of output. Actions are restricted to the set of integers from 1 to $n$. Let $e_{1}, \ldots, e_{N}$ denote the actions taken by $N$ players. The payoff function for player $i$ is given by:

$$
\begin{gathered}
\pi\left(e_{i}, e_{\min }\right)=a e_{\min }-b e_{i} \\
a>b>0,
\end{gathered}
$$

where $e_{\min }$ equals $\min \left(e_{1}, \ldots e_{N}\right)$. Thus each player's payoff increases in the minimum level of effort contributed to the team production, and decreases in player's own level of effort.

The best response for each player is to take the level of effort, $e_{i}$ equal to $e_{\min }$. Thus, all feasible actions are potential Nash equilibrium outcomes which can be Pareto ranked. The payoff-dominant equilibrium is the one in which each player chooses the maximum level of effort, $n$. The equilibrium with the lowest payoff to each player is the one in which everyone chooses the least amount of effort, $e_{i}=1$

\footnotetext{
${ }^{3}$ See Cooper (1999) for a good discussion of the importance of team-production games for modeling the role of agents' expectations in macroeconomic models with strategic complementarities.
} 
for all $i$. For the set of payoff parameter values, if a selection principle is based on the riskiness of an equilibrium point, security selects the equilibrium with maximin actions, i.e. the equilibrium with the lowest payoff. The payoff-dominant selection principle, on the other hand, chooses an equilibrium with the maximum level of effort, $n$, for each player $i, i \in[1, N]$.

\subsection{EXPERIMENTS WITH HUMAN SUBJECTS}

The results of Van Huyck et al. experiments with human subjects showed the following. The treatments with large groups (group size equal to 14 and 15) converged to the maximin (minimum effort) equilibrium actions. In the large group treatments in which $b$ was set to zero, participants coordinated on the payoff-dominant equilibrium.

The treatments with small groups where the group size was equal to 2 resulted in the payoff-dominant equilibrium for the cases where pairs were fixed for the duration of the experiment. The outcome was indeterminate in those cases where players were randomly matched in each iteration of the game. These experimental results suggest that the large group size does introduce the amount of strategic uncertainty into the game sufficient enough to result in a selection of the minimum effort equilibrium and thus in a coordination failure. In addition, the payoff dominant equilibrium result observed in small group treatments suggests that the group size plays important role in coordination problems.

Following this experimental work, Berninghaus and Ehrhart (1998) showed that the number of repetitions of the game in the experiments with human subjects was another important determinant for equilibrium selection. If the game is repeated large number of times, the payoff dominant outcome can be observed even in games with many players (group size equal to 6). By sufficiently reducing the number of repetitions, Berninghaus and Ehrhart were able to reproduce Van Huyck et al. experimental results for large groups.

\subsection{Evolutionary GAME THEORY AND ADAPTIVE TECHNIQUES}

As mentioned in the introduction, several papers have examined the behavior of evolutionary and adaptive models in Van Huyck et al. environment. However, they all analyzed versions of the game with large group treatment (Crawford, 1991, 1995; Roth, 1995) and random-pairing treatment (Crawford, 1991). None of the studies modeled the version of the game with fixed-pairing.

Crawford (1991) studies evolutionary stability of equilibria of Van Huyck et al. stage game. He shows that, in large or finite populations, the strategy configuration in which all players choose the lowest effort is the unique evolutionarily stable strategy (for $b>0)^{4}$. For $b=0$, there is no evolutionarily stable strategy.

\footnotetext{
${ }^{4}$ Lower efforts always yield the same output shares as higher efforts, but at lower cost. Thus, in any population, large or finite, a mutation to an effort that is the minimum in the population then has a higher payoff than all other efforts.
} 
Crawford's random-pairing treatment results show that any pure strategy effort is monomorphic evolutionary stable strategy whenever a number of players, $n$, is greater than 4 . For $n=2$ and $n=3$, he shows that the only evolutionary stable strategy is the one with the lowest effort.

Crawford (1995) discusses the results of the analysis when the methods of Kandori et al. (1993) and Young (1993) are applied to the model. In the long run, the process cycles perpetually among the pure-strategy equilibria of the underlying game, with their prior probabilities at any given time determined by the ergodic distribution. As the level of noise is allowed to approach zero, the ergodic distribution assigns the probability of one of approaching the equilibrium with the lowest effort.

Crawford (1995) follows the adaptive control literature in assuming that players' beliefs and strategy choices evolve according to a linear adjustment rule. On average, beliefs adjust part of the way toward the value suggested by the latest observation of the minimum effort. These average adjustments are perturbed each period by idiosyncratic shocks that bring strategic uncertainty into the model. For large group treatments, the model reproduces the dynamics observed in the experimental data and provides an adequate statistical summary of the subjects' behavior.

Finally, Roth (1995) describes the application of the reinforcement algorithm to the Van Huyck et al. game. The common learning version of the algorithm converges to the minimum effort equilibrium.

All of the above mentioned applications of the evolutionary game theory analysis and of the adaptive algorithms select the minimum effort equilibrium. These applications are successful in explaining the behavior observed in the large group experimental treatments. However, they do not address the fixed pairs experimental treatment in which convergence to the payoff dominant equilibrium was observed. An exception is Arifovic (1996b) that demonstrated that the populations of players that used the genetic algorithm to update their strategies coordinated on the high effort equilibrium in case of the group size equal to 2 , and on the low effort equilibrium in case of the group size equal to 14.

All of the above studies are based on the assumption that players play repeatedly a stage game. Thus, stage-game strategies are the objects of choice that players adjust over time. Crawford (1991) notes that, in principle, the learning in coordination games with strategic uncertainty, can be studied within the context of repeated games. However, the multiple-equilibrium problem does not get resolved in repeated games. In fact, the problem becomes more difficult since repeated game approach requires that players' expectations are coordinated at the start of a play, at an equilibrium selected by applying refinements that are quite insensitive to strategic uncertainty ${ }^{5}$.

\footnotetext{
${ }^{5}$ Crawford does note that repeated-game strategies are essential in fixed-pairs treatment and were recognized by Van Huyck et al. subjects.
} 


\section{RepeAted GAME}

A population of $M$ players participate in a repeated team-production game. Players have identical payoff functions and strategy sets. The time is divided into epochs. At the beginning of each epoch, $h \in\left\{1, \ldots, h_{\max }\right\}$, players are randomly placed into groups of size $N$. They play the game within a given group for $T+1$ periods. At the end of an epoch, $h$, players are randomly placed into new groups of size $N$ and the new epoch, $h+1$, begins.

Players' strategies are described in the following way. A strategy $s_{i}, i \in\{1, M\}$, is given by a vector of values:

$$
\left[p_{0}, p_{1}, p_{2}, \ldots, p_{n}\right]
$$

The value of $p_{0}$ indicates the level of effort that a player plays at the initial time period $t=0$ of an epoch, and $p_{j}$ indicates the level of effort that the player plays at $t$ given that the minimum group effort at time $t-1$ was $j$. Suppose that $n=4$ and that player's strategy at epoch $h$ is given by:

\begin{tabular}{ccccc}
\hline$p_{0}$ & $p_{1}$ & $p_{2}$ & $p_{3}$ & $p_{4}$ \\
\hline 3 & 2 & 4 & 1 & 4 \\
\hline
\end{tabular}

This strategy has the following interpretation. A player plays $e_{0}=3$ at $t=0$ of a given epoch. In subsequent iterations, $t>0$, she plays $e_{t}=2$ if $e_{\min , t-1}=1$, $e_{t}=4$ if $e_{\min , t-1}=2, e_{t}=1$ if $e_{\min , t-1}=3$, and $e_{t}=4$ if $e_{\min , t-1}=4$. At the end of each iteration $t, t \in\{0, \ldots, T\}$, players payoffs are computed, and they are averaged at the end of each epoch. (The discount factor is equal to 1.)

There are two types of Nash equilibria of this game, type $s$ stationary equilibria, and type $c$ cyclical equilibria. Denote the set of stationary, $s$, equilibria as $E_{s}$. This set contains two subsets, one that we call the full scale stationary equilibria, $E_{s}^{f}$, and the other that we call the limited scale stationary equilibria, $E_{s}^{l}$.

In the full scale equilibria, a level of effort $i, i \in\{1, \ldots, n\}$ is played for the entire duration of an epoch $h$, i.e. for $T+1$ iterations. The number of equilibria in $E_{s}^{f}$ is equal to the number of Nash equilibria of the original one-shot game described in section 2, i.e. it is equal to the number of possible levels of effort, $n$. In this subset, a strategy $s_{i}^{*}$ is a Nash equilibrium strategy with the level of effort $i$ if it induces a level of effort $i$ at the initial position, i.e. $p_{0}=i$ at $t=0$, and a level of effort $i$ at a position $p_{i}$, i.e. $p_{i}=i$ for $t \geq 0$. This strategy is a best response to itself.

Suppose that the entire population of players plays strategy $s_{i}^{*}$. At $t=0$, within each group $r$, each player contributes a level of effort $i$ since they all have $p_{0}$ equal to $i$. Thus, the minimum level of effort at $t=0$ is $i$. Then, at $t=1$, given that $e_{\min , 0}=i$, each player takes an action prescribed by the $i^{t h}$ position, $p_{i}$. For all players, $p_{i}=i$, and thus all of them (within their groups) contribute the level of effort $i$ which is the minimum level of effort at $t=1$. For all $t>1$, players continue taking the action prescribed by $p_{i}$. The resulting payoff for a strategy $s_{i}^{*}$ in every time period is equal to: $\pi_{i}=a i-b i$. 
However, the strategy $s_{i}^{*}$ is not the unique best response to itself. Any strategy $s_{k}, k \in\{1, \ldots, M\}$, such that $p_{0}=i$ and $p_{i}=i$ is a best response to $s_{i}^{*}$ and is also a Nash equilibrium strategy. This strategy can have different levels of effort at some or all positions $p_{j}, j \neq i$. Thus, $p_{0}\left(s_{k}\right)=p_{0}\left(s_{i}^{*}\right)=i, p_{i}\left(s_{k}\right)=p_{i}\left(s^{*}\right)=i$, and $p_{j}\left(s_{k}\right) \neq p_{j}\left(s^{*}\right)$ for some $j \neq i, j \in\{1, \ldots, n\}$. Thus, for each level of effort $i$, there is a subset of full scale stationary equilibria, $E_{i, s}^{f}$, whose elements are strategies that are best responses to $s_{i}^{*}$, i.e.

$$
E_{i, s}^{f}=\left\{s_{k}=\left[p_{0}, p_{1}, \ldots, p_{n} \mid p_{0}=i, p_{i}=i\right]\right\} .
$$

All strategies in this set are Nash equilibrium strategies for the level of effort $i$. Since $s_{i}^{*}$ is not a unique best response to itself, Nash equilibria of this type are weak. This fact is important for the behavior of the evolutionary model of this game over time.

The set of the limited scale stationary equilibria contains equilibria where, in each $h$, non-stationary values are played for the initial $T_{\text {init }} \leq n-1$ iterations and stationary value $e_{t}=i$ is played for all the remaining iterations $t$, such that $T_{\text {init }}<t \leq T$. Thus, with equilibrium strategy $s_{i}^{*}$, for $0 \leq t \leq T_{\text {init }}$, players choose levels of effort from positions $p_{j}$ such that $p_{j}=k$ where $k \neq j$, and starting with $t>T_{\text {init }}$, they play $p_{i}=i$ for the remaining iterations. Consider the following limited scale stationary equilibrium strategy with $n=4$ :

\begin{tabular}{ccccc}
\hline$p_{0}$ & $p_{1}$ & $p_{2}$ & $p_{3}$ & $p_{4}$ \\
\hline 1 & 4 & 2 & 2 & 4 \\
\hline
\end{tabular}

At $t=0$, a player $i, i \in\{1, \ldots, M\}$, plays $e_{0}=1$, at $t=1$, she plays $e_{1}=4$, and for subsequent $t>1$, she plays $e_{t}=4$. Here $T_{\text {init }}=1$.

Again, an equilibrium strategy $s_{i}^{*}$ is not the unique best response to itself. Any strategy $s_{k} \neq s_{i}^{*}$ that induces the same non-stationary values of effort for the initial number of iterations $T_{\text {init }}<n-1$ and a stationary value of effort $i$ for the remaining iterations is a best response to $s_{i}^{*}$. However, if $T_{\text {init }}=n-1$, strategy $s_{i}^{*}$ is the unique best response to itself. This happens because all of the positions of the strategy participate in inducing an equilibrium level of effort and thus, the value of each position matters for the play of the equilibrium sequence. Thus, the subset of limited scale stationary equilibria with stationary value of effort $i$, is given by:

$$
\begin{gathered}
E_{i, s}^{l}=\left\{s \mid \exists i_{1} \neq i_{2} \ldots \neq i_{T_{i n i t}} \text { s.t } s_{k}=\left[p_{0}, p_{1}, \ldots, p_{n}\right] \text { with } p_{0}=i_{1},\right. \\
\left.p_{i_{k}}=i_{k+1}, 1 \leq k \leq T_{\text {init }}, p_{T_{\text {init }}}=i, p_{i}=i \forall k\right\}
\end{gathered}
$$

All of the limited scale equilibria with $T_{\text {init }}<n-1$ are weak. The only strict limited scale equilibrium is the one where $T_{\text {init }}=n-1$.

The second set of equilibria are the cyclical equilibria. Denote the set of cyclical equilibria by $E_{c}$. These cyclical equilibria can be of order 2 up to order $n$. Consider 
the following example:

\begin{tabular}{ccccc}
\hline$p_{0}$ & $p_{1}$ & $p_{2}$ & $p_{3}$ & $p_{4}$ \\
\hline 2 & 4 & 4 & 2 & 2
\end{tabular}

The above is an illustration of the equilibrium strategy of cycle of order 2 . At $t=0$, a player plays $e_{0}=2$. Then, at $t=1$, she plays $e_{1}=4$, and at $t=2$, she plays $e_{2}=2$. After this, she plays $e_{t}=4$ at every odd $t$, and $e_{t}=2$ at every even $t$.

Suppose now that $n=8$. Then the following are the cyclical equilibria of the game:

Equilibria of order 2:

$p_{0}=i, p_{i}=j, p_{j}=i$

Equilibria of order 3 :

$p_{0}=i, p_{i}=j, p_{j}=k, p_{k}=i$

Equilibria of order 4 :

$p_{0}=i, p_{i}=j, p_{j}=k, p_{k}=l, p_{l}=m$

Equilibria of order 5:

$p_{0}=i, p_{i}=j, p_{j}=k, p_{k}=l, p_{l}=m, p_{m}=i$

Equilibria of order 6 :

$p_{0}=i, p_{i}=j, p_{j}=k, p_{k}=l, p_{l}=m, p_{m}=o, p_{o}=i$

Equilibria of order 7:

$p_{0}=i, p_{i}=j, p_{j}=k, p_{k}=l, p_{l}=m, p_{m}=o, p_{o}=p, p_{p}=i$

Equilibria of order 8:

$p_{0}=i, p_{i}=j, p_{j}=k, p_{k}=l, p_{l}=m, p_{m}=o, p_{o}=p, p_{p}=q, p_{q}=i$

Any cyclical equilibrium strategy of order less than $n$ is not the unique best response to itself. Thus, all of the cyclical equilibria of order 2 to $n-1$ are weak. The cyclical equilibrium of order $n$ is a strict Nash equilibrium because an equilibrium strategy of a cycle of order $n$ is the unique best response to itself.

\subsection{Monomorphic POpulation With PAirWise MATCHing}

The analysis of the evolutionary stability of the Nash equilibrium strategies is carried out for the group of size 2, and for the monomorphic equilibria, i.e. equilibria in which all players in the population use the same strategy. Consider a population with an infinite number of players who are matched randomly pairwise.

A strategy $s_{i}$ is an evolutionary stable strategy (ESS) if, for all strategies $s_{j} \neq s_{i}$, and $\delta$ sufficiently small,

$$
\delta \pi\left(s_{i}, s_{j}\right)+(1-\delta) \pi\left(s_{i}, s_{i}\right)>\delta \pi\left(s_{j}, s_{j}\right)+(1-\delta) \pi\left(s_{j}, s_{i}\right) .
$$

If the above condition is satisfied, a population of players in a monomorphic equilibrium cannot be successfully invaded by a small fraction of players using a different strategy. If the invasion is to fail, resident players must get a strictly larger expected payoff than invaders at the time when invasion occurs.

A Nash equilibrium strategy $s^{*}$ is stable with respect to any strategy that is not a best response to $s^{*}$. However, $s^{*}$ is not stable with respect to $s_{j} \neq s^{*}$ that is 
a best response to $s^{*}$. From here, it follows that strategy $s_{i}^{*}$ is not ESS since if all players are using $s_{i}^{*}$, an invasion of players using $s_{j}$ that is best response to $s^{*}$ will not be driven out (nor will they necessarily come to dominate the population). The only Nash equilibria that are evolutionary stable are limited scale stationary equilibria with $T_{\text {init }}=n-1$, and cyclical equilibria of order $n$.

Neutral stability is a stability concept that is weaker than the concept of evolutionary stability. A strategy $s_{i}$ is a neutrally stable strategy (NSS) if, for all strategies $s_{j} \neq s_{i}$,

$$
\delta \pi\left(s_{i}, s_{j}\right)+(1-\delta) \pi\left(s_{i}, s_{i}\right) \geq \delta \pi\left(s_{j}, s_{j}\right)+(1-\delta) \pi\left(s_{j}, s_{i}\right) .
$$

While evolutionary stability requires that no mutant strategy persists, in the sense of earning an equal or higher payoff, neutral stability requires that no mutant thrives in the sense of earning a higher payoff than the incumbent strategy (Weibull, 1995) ${ }^{6}$.

All of the Nash equilibria of the game with infinite number of players and random matching are neutrally stable. For limited scale stationary equilibria with $T_{\text {init }}=n-1$ and cycle of order $n$ the above condition holds as a strict inequality. For other equilibria it holds as a strict inequality for strategies that are not best response to equilibrium strategies, but holds with equality for those strategies that are best responses ${ }^{7}$.

The neutral stability of Nash equilibria implies that a fraction of invaders will not necessarily disappear from the population. This, in turn, can result in switching of the population of strategies between different equilibria.

Thus, the results of the analysis of the Nash equilibria of the repeated game with polymorphic populations and of the evolutionary stability analysis of the equilibria with monorphic populations provide intuition as to why the genetic algorithm populations of agents move through different equilibria.

\section{The GENETIC ALGORITHM APPLICATION}

Our boundedly rational players are endowed with strategies for a repeated game. They are aware of the repeated character of the game, but are not fully rational as to coordinate their expectations from the start of the repeated game.

Players' decision rules are represented by a population of $M$ binary strings. At each epoch $h, h \in\left\{1, \ldots, h_{\max }\right\}$, players are randomly placed into groups of

\footnotetext{
${ }^{6}$ Note that a set of evolutionary stable strategies is a subset of a set of neutrally stable strategies.

${ }^{7}$ Eaton and Morrison (1998) provide a nice illustration of the effects of interaction between neutral stability of equilibria and genetic drift. They study an evolutionary model of a leader/follower game. The subgame perfect Nash equilibrium of the game in which retaliation does not occur is inefficient. However, evolutionary stability does not rule out equilibria which involve retaliation because the equilibria of the game are neutrally stable. Computer simulations of the system oscillate between equilibria that include retaliatory behavior and equilibria which do not.
} 
size $g$. Within each group, players repeatedly play the game for $T$ iterations. A player $i$ uses a binary string $i$ to make a decision about her action at each iteration, $t, t \in\{0, \ldots, T\}$. A binary string consists of $n+1$ positions where $n=8$ is the maximum feasible action. Each position $p_{j}, j \in\{0, \ldots, n\}$, consists of $l$ bits where $l$ is the number of bits required to encode integer value $n$. The total length of a string is $(n+1) l$ bits. The first position, $p_{0}$, encodes the action that player takes at iteration $t=0$. A position $p_{j}, j \in[1, n]$ encodes the action that player $i$ takes at iteration $t>0$, given that a minimum effort of her group at iteration $t-1, e_{t-1, \min }$, was equal to $j$. An example of a binary string that encodes integer actions in the interval $\{1, \ldots, 8\}$ is given below:

$\begin{array}{lccccccccc}\text { position } p_{j} & 0 & 1 & 2 & 3 & 4 & 5 & 6 & 7 & 8 \\ \text { binary string: } & 000 & 101 & 001 & 110 & 011 & 111 & 000 & 011 & 100 \\ \text { action } e_{i, 0} & 1 & & & & & & & & \\ \text { action } e_{i, t}: & & 6 & 2 & 7 & 3 & 8 & 1 & 4 & 5 \\ \bar{e}_{t-1, \min } & & 1 & 2 & 3 & 4 & 5 & 6 & 7 & 8\end{array}$

The strategy encoded by this binary string is interpreted in the following way. At iteration $t=0$, player $i$ takes action $e_{i, 0}=1$. For $t>1$, the player decides on effort 6 at iteration $t$ if $e_{t-1, \min }$ was equal to 1 ; the player decides on effort 2 at $t$ if $e_{t-1, \min }$ was equal to 2 ; and so on.

The algorithm is implemented in the following way:

1. At each epoch $h$, players are randomly placed into groups of size $N$. Each player's strategy is represented by a binary string. The size of the population of binary strings, $M$, divided by $N$, the group size, determines the number of groups $g$.

2. Within each group, the players play the game that is repeated for $T+1$ iterations.

3. Each player $i, i \in\{1, \ldots, M\}$, chooses her action. Initial action, at $t=0$, is determined by the position $p_{0}$. For $t>0, t \in\{1, \ldots, T\}$, each player chooses her action at $t$ by using the information about, $e_{t-1, \min }$, the minimum group effort from the previous, $t-1$, iteration. Given $e_{t-1, \min }=j, j \in\{1, \ldots, n\}$, the player looks up the $j^{\text {th }}$ position of her binary string and decodes the action that she takes at $t$.

4. Once each player chooses her action for iteration $t$, the minimum group effort of the current iteration is determined within each group.

5. The payoff for each player is computed.

6. The payoffs are accumulated during $T+1$ iterations of an epoch $h$. The average payoff for each player $i$ is computed as $\sum_{t=0}^{T} \pi_{i, t} /(T+1)$.

7. At the end of epoch $h$, players' strategies are updated using the genetic operators: reproduction, crossover and mutation. The updating results in a new population of binary strings that are used in epoch $h+1$.

8. Steps 2 through 8 are repeated for $h_{\max }$ epochs.

9. Initially, at $h=1$, a binary string for each player is chosen randomly. 


\subsection{Description of genetic operators}

The reproduction operator makes copies of individual binary strings. The criterion used in copying is the value of the fitness function. The fitness of a string $i$ at epoch $h, \mu_{i, h}$, is equal to the average payoff that player $i$ earned over $T+1$ iterations,

$$
\mu_{i, h}=\frac{\sum_{t=0}^{T} \pi_{i, t}}{T+1} .
$$

We use a tournament selection method to generate a population of $M$ copies of binary strings. It is implemented in the following way. Two binary strings are randomly selected and their fitnesses are compared. The binary string with a higher fitness value is copied and placed into the mating pool. Tournament selection is repeated $M$ times so as to maintain a constant population of size $M$. Thus, $M$ copies of the decision rules that have been shown to be relatively more successful than other rules enter into a "mating pool". The purpose of reproduction is to promote well performing strings over time by assigning increasing number of copies of the strings with the above the average fitness values ${ }^{8}$.

The crossover operates on the pairs of randomly selected binary strings. First, two binary strings are selected from the mating pool at random. Secondly, a number $k, k \in\{1, \ldots, l-1\}$, is randomly selected and two new binary strings are obtained by swapping the bit values to the right of the position $k$. A total of $M / 2$ pairs (where $M$ is even integer) are selected. Crossover takes place with probability $p_{\text {cross }}$.

The mutation operator randomly changes the value of a position within a binary string. Each position has a probability $p_{m u t}$ of being altered by mutation, independent of other positions.

Given the updated set of binary strings, the algorithm proceeds to stage 2 . Each simulation is conducted for a total of $h_{\max }$ epochs. Initial population of binary strings is randomly generated.

\section{THE RESUlTS}

In order to examine the effect of different group sizes on the behavior of the model, simulations were conducted for $N=2,3,4,5,6,7$ and 14 . The values of the payoff parameters were: $a=1.2$ and $b=0.6$. The following genetic algorithm parameter values were used: probability of crossover, $p_{\text {cross }}=0.6$, probability of mutation, $p_{m u t}=0.0033$, population size, $\mathrm{M}=40$ or 42 , number of epochs, $h_{\max }=100,000$, and the number of iterations within an epoch, $T=50$. The large number of epochs was used in order to examine the long-run behavior of the model.

\footnotetext{
${ }^{8}$ Tournament selection has been shown in the literature on evolutionary computation (see Back, 1996) to have better properties than the roulette wheel type of selection operator. However, within the model described in this paper, implementation of either of these operators generates very similar dynamics. Results of simulations in which roulette wheel was used are available from the author upon request.
} 
TABLE 1. Frequency distribution of average effort over 100,000 epochs.

\begin{tabular}{lcccccccc}
\hline $\begin{array}{l}\mathrm{e} \\
\text { frequency }\end{array}$ & 1 & 2 & 3 & 4 & 5 & 6 & 7 & 8 \\
$\mathrm{~g}=2$ & 0.00 & 0.00 & 0.00 & 0.00 & 0.00 & 0.02 & 0.14 & 0.84 \\
$\mathrm{~g}=3$ & 0.00 & 0.00 & 0.00 & 0.00 & 0.05 & 0.15 & 0.37 & 0.43 \\
$\mathrm{~g}=4$ & 0.00 & 0.00 & 0.00 & 0.01 & 0.08 & 0.15 & 0.44 & 0.32 \\
$\mathrm{~g}=5$ & 0.00 & 0.00 & 0.01 & 0.06 & 0.18 & 0.29 & 0.25 & 0.21 \\
$\mathrm{~g}=6$ & 0.00 & 0.02 & 0.04 & 0.13 & 0.19 & 0.30 & 0.23 & 0.1 \\
$\mathrm{~g}=7$ & 0.00 & 0.01 & 0.04 & 0.17 & 0.24 & 0.30 & 0.16 & 0.08 \\
$\mathrm{~g}=8$ & 0.00 & 0.03 & 0.07 & 0.20 & 0.19 & 0.29 & 0.18 & 0.04 \\
$\mathrm{~g}=10$ & 0.00 & 0.11 & 0.13 & 0.24 & 0.23 & 0.23 & 0.04 & 0.02 \\
$\mathrm{~g}=12$ & 0.00 & 0.17 & 0.16 & 0.21 & 0.18 & 0.23 & 0.04 & 0.01 \\
$\mathrm{~g}=14$ & 0.00 & 0.34 & 0.20 & 0.20 & 0.10 & 0.13 & 0.02 & 0.01 \\
\hline
\end{tabular}

TABLE 2. Frequency distribution of average minimum effort over 100,000 epochs.

\begin{tabular}{lcccccccc}
\hline $\begin{array}{l}\mathrm{e} \\
\text { frequency }\end{array}$ & 1 & 2 & 3 & 4 & 5 & 6 & 7 & 8 \\
$\mathrm{~g}=2$ & 0.00 & 0.00 & 0.00 & 0.00 & 0.00 & 0.4 & 0.27 & 0.69 \\
$\mathrm{~g}=3$ & 0.00 & 0.00 & 0.00 & 0.01 & 0.1 & 0.13 & 0.4 & 0.36 \\
$\mathrm{~g}=4$ & 0.00 & 0.00 & 0.00 & 0.07 & 0.12 & 0.16 & 0.46 & 0.19 \\
$\mathrm{~g}=5$ & 0.00 & 0.00 & 0.04 & 0.06 & 0.31 & 020 & 0.26 & 0.13 \\
$\mathrm{~g}=6$ & 0.00 & 0.02 & 0.04 & 0.20 & 0.30 & 0.18 & 0.19 & 0.07 \\
$\mathrm{~g}=7$ & 0.01 & 0.01 & 0.12 & 0.16 & 0.35 & 0.16 & 0.17 & 0.03 \\
$\mathrm{~g}=8$ & 0.00 & 0.06 & 0.16 & 0.17 & 0.35 & 0.15 & 0.10 & 0.01 \\
$\mathrm{~g}=10$ & 0.05 & 0.11 & 0.24 & 0.20 & 0.31 & 0.06 & 0.03 & 0.00 \\
$\mathrm{~g}=12$ & 0.07 & 0.17 & 0.23 & 0.15 & 0.29 & 0.07 & 0.02 & 0.00 \\
$\mathrm{~g}=14$ & 0.18 & 0.25 & 0.23 & 0.14 & 0.15 & 0.04 & 0.01 & 0.00 \\
\hline
\end{tabular}

The results of simulations are summarized in tables 1 and 2 . Table 1 presents the frequency distribution of the values of effort averaged over $T+1$ iterations of each epoch, $\bar{e}_{h}$ :

$$
\bar{e}_{h}=\frac{\sum_{t=0}^{T} \sum_{i=1}^{M} e_{i, t}}{M(T+1)} .
$$

The value of the average effort calculated in this way represents a data point in the frequency distribution. All the values of $\bar{e}_{h}$ are truncated to the nearest smaller integer $i$. Table 2 shows the frequency distribution of the average values of minimum effort, $\bar{e}_{\min , h}$, calculated in the following way:

$$
\bar{e}_{m i n, h}=\frac{\sum_{i=1}^{N} \sum_{t=0}^{T} e_{m i n, j, t}}{N(T+1)} .
$$


TABLE 3. Frequency distribution of best response measure over 100,000 epochs.

\begin{tabular}{lcccccccccc}
\hline $\begin{array}{l}\text { interval } \\
\text { frequency }\end{array}$ & 0.1 & 0.2 & 0.3 & 0.4 & 0.5 & 0.6 & 0.7 & 0.8 & 0.9 & 1 \\
$\mathrm{~g}=2$ & 0.00 & 0.00 & 0.00 & 0.00 & 0.00 & 0.01 & 0.02 & 0.04 & 0.16 & 0.77 \\
$\mathrm{~g}=3$ & 0.00 & 0.00 & 0.00 & 0.00 & 0.00 & 0.00 & 0.01 & 0.04 & 0.15 & 0.80 \\
$\mathrm{~g}=4$ & 0.00 & 0.00 & 0.00 & 0.00 & 0.00 & 0.01 & 0.02 & 0.07 & 0.22 & 0.68 \\
$\mathrm{~g}=5$ & 0.00 & 0.00 & 0.00 & 0.00 & 0.00 & 0.01 & 0.03 & 0.08 & 0.23 & 0.64 \\
$\mathrm{~g}=6$ & 0.00 & 0.00 & 0.00 & 0.00 & 0.00 & 0.01 & 0.03 & 0.09 & 0.24 & 0.62 \\
$\mathrm{~g}=7$ & 0.00 & 0.00 & 0.00 & 0.00 & 0.01 & 0.02 & 0.04 & 0.10 & 0.23 & 0.60 \\
$\mathrm{~g}=8$ & 0.00 & 0.00 & 0.00 & 0.00 & 0.01 & 0.02 & 0.04 & 0.08 & 0.20 & 0.65 \\
$\mathrm{~g}=10$ & 0.00 & 0.00 & 0.00 & 0.01 & 0.01 & 0.02 & 0.03 & 0.09 & 0.15 & 0.70 \\
$\mathrm{~g}=12$ & 0.00 & 0.00 & 0.00 & 0.00 & 0.01 & 0.02 & 0.03 & 0.07 & 0.13 & 0.75 \\
$\mathrm{~g}=14$ & 0.00 & 0.00 & 0.00 & 0.01 & 0.01 & 0.01 & 0.03 & 0.04 & 0.08 & 0.81 \\
\hline
\end{tabular}

where $e_{\min , j, t}$ is the minimum effort in group $j, j \in\{1, \ldots, N\}$. The results clearly show that the frequencies of high values of $\bar{e}_{h}$ and $\bar{e}_{m i n, h}$ decrease with increases in the group size. For example, populations with $N=2$ spend most of the time playing $e=8$, populations with $N=3$ spend most of the time playing $e=7$, etc.

At the same time, the common feature of the behavior observed in simulations with all group sizes is that a large fraction of genetic algorithm populations spends most of the time playing best response actions. This feature is present regardless of the group size. In order to examine how close an entire population of strategies was to playing best response actions at each epoch, the best response measure was calculated in the following way:

$$
b r_{h}=\frac{\sum_{t=0}^{T} \sum_{i=1}^{M} I_{i, t}}{M(T+1)},
$$

where $I_{i, t}$ is an index variable equal to 1 when player's $i$ effort at iteration $t$ is equal to the minimum within the group in which she plays, and 0 if it is greater than the minimum effort. Frequency distribution of this measure for all group sizes is given in Table 3 .

The frequency distribution of $b r_{h}$ shows that, regardless of the group size, the mass of the distribution is concentrated on the values of the measure close to one. This means that, for all group sizes, the genetic algorithm populations spend most of the time close to the Nash equilibria. Lower values of the best response measure occur only during brief periods of time during which the transition from one equilibrium to the other takes place.

In order to understand the dynamics of the evolutionary process, it is helpful to consider the following hypothetical example. Suppose that players are playing in groups of size $N=2$. All players' strategies are such that they choose initial level of effort equal to $1, p_{0}=1$ for all $i$, and that they have $p_{1}=1$. Thus, all players choose best response actions and the economy is in the minimum effort 


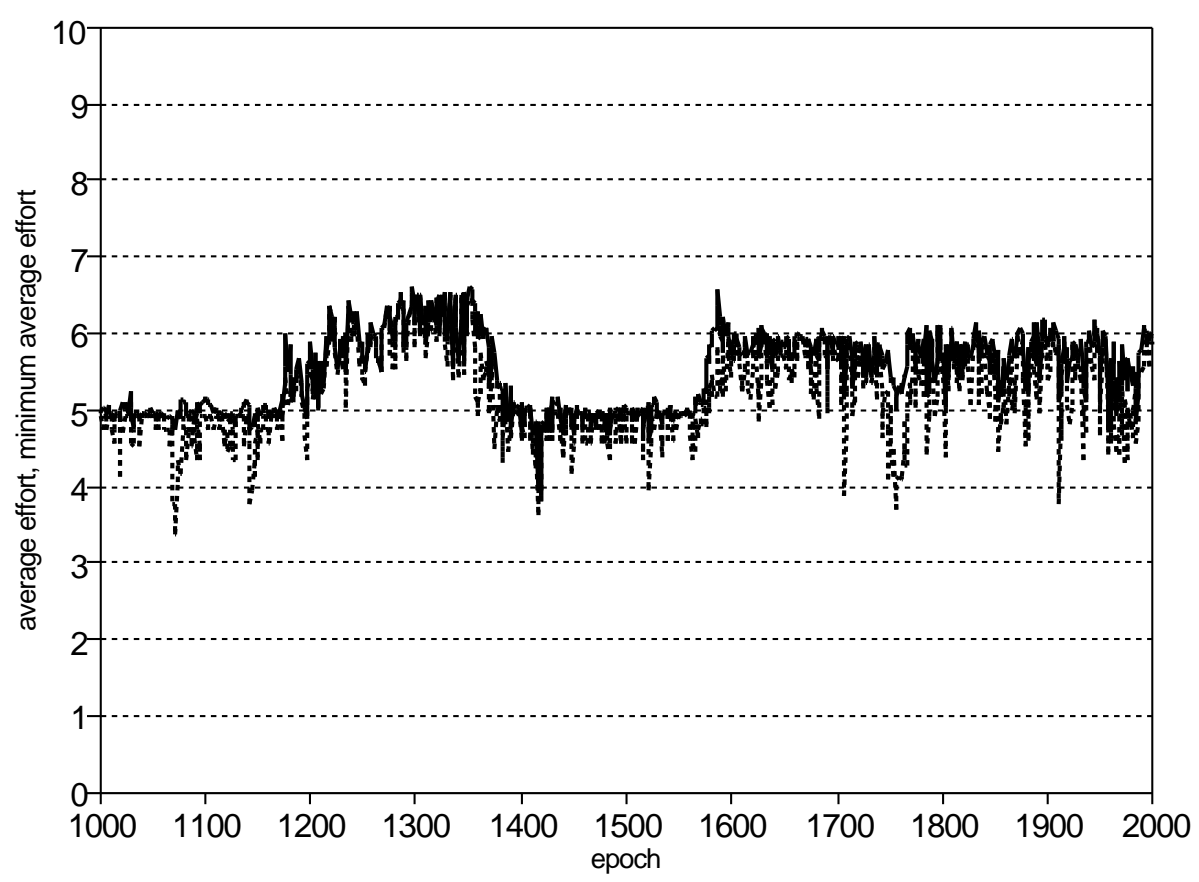

Figure 1. Average and average minimum effort; group size $=4$.

Nash equilibrium. A positive rate of mutation will result in small deviations from the best response, but strategies prescribing actions $p_{1}=k, k>1$, at location 1 will be eliminated from the population since they will have lower average payoff than the strategies with $p_{1}=1$. At the same time, mutation will result in changes in other parts of players' strategies, not currently used in the game. Over time, mutation will generate a number of strings with strategies $s^{\prime}$ such that $p_{0}=1$ and $p_{1}=1$, but in addition $p_{j}=j$ at some $j>1$. These changes will be irrelevant for the levels of effort that are actually chosen. Eventually positions that encode initial levels of effort within these strings will also be affected by mutation, i.e. mutation will result in the initial levels of effort equal to $j$. When a sufficient number of such strings is generated at the same time, they will earn higher average payoffs than the strings that encode $p_{0}=1$. Reproduction will promote these strings and they will overtake the population. This process works in the reverse as well, with population going from the higher to the lower level of effort.

Another interesting feature of the observed dynamics is the protective role played by the positions $p_{j} \neq i$ during the periods when the equilibrium level of effort $i$ is played. Frequently, a number of positions $p_{j} \neq p_{i}$ encode the value of effort $i$. This works as a protection of the population against the invasion of mutants and contributes to the efficient playing, i.e. mainting high values of the measure of best response. 


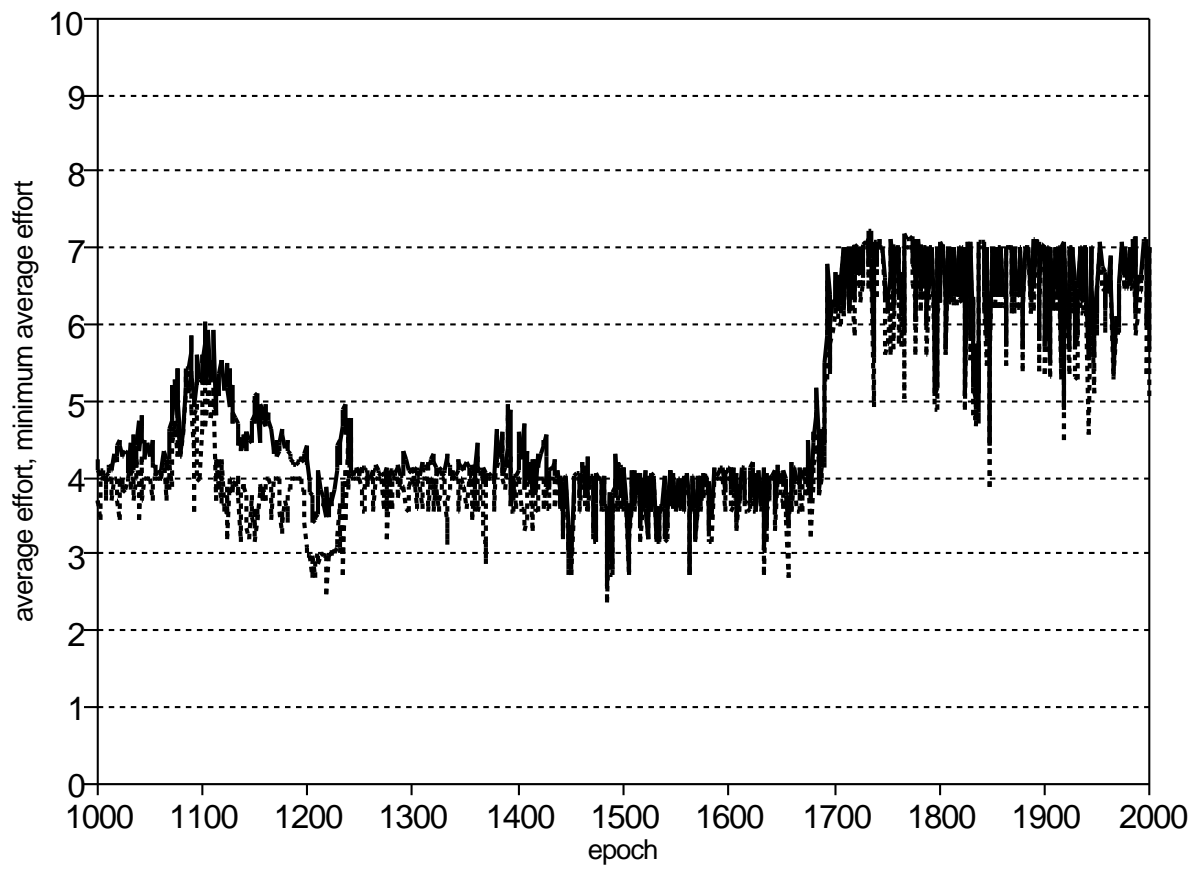

Figure 2. Average and average minimum effort; group size $=6$.

Figures 1-4 show the behavior of the average effort and average minimum effort over a thousand epochs for group sizes $g=4, g=6, g=10$ and $g=14$.

Table 4 is an illustration of a population of strategies at $h=500$ in a simulation with $g=6$. The population is playing a stationary equilibrium with $p_{0}=5$ and $p_{5}=5$. (To save space, only 30 out of 42 strategies are presented.) Notice that positions $p_{2}$ and $p_{6}$ also contain a number of 5 's that serve as a protection of the stationary equilibrium with the level of effort $i=5$ against the mutants. Table 5 shows the population at $h=1300$ of the same simulation. Now, the population is playing a cycle of order 2 , with $p_{3}=8$ and $p_{8}=3$.

The frequency of occurrence of cycles requires further investigation and exceeds the scope of this paper. In the initial simulations, cycles of order 2 and 4 were observed with frequency that was low relatively to occurrence of stationary equilibria.

What happens if we change the rate of mutation, crossover or the payoff parameter values? Increase in the rate of mutation $\left(p_{m u t}=0.033\right)$ resulted in the dynamics with relatively low levels of effort even for small group sizes. The above 


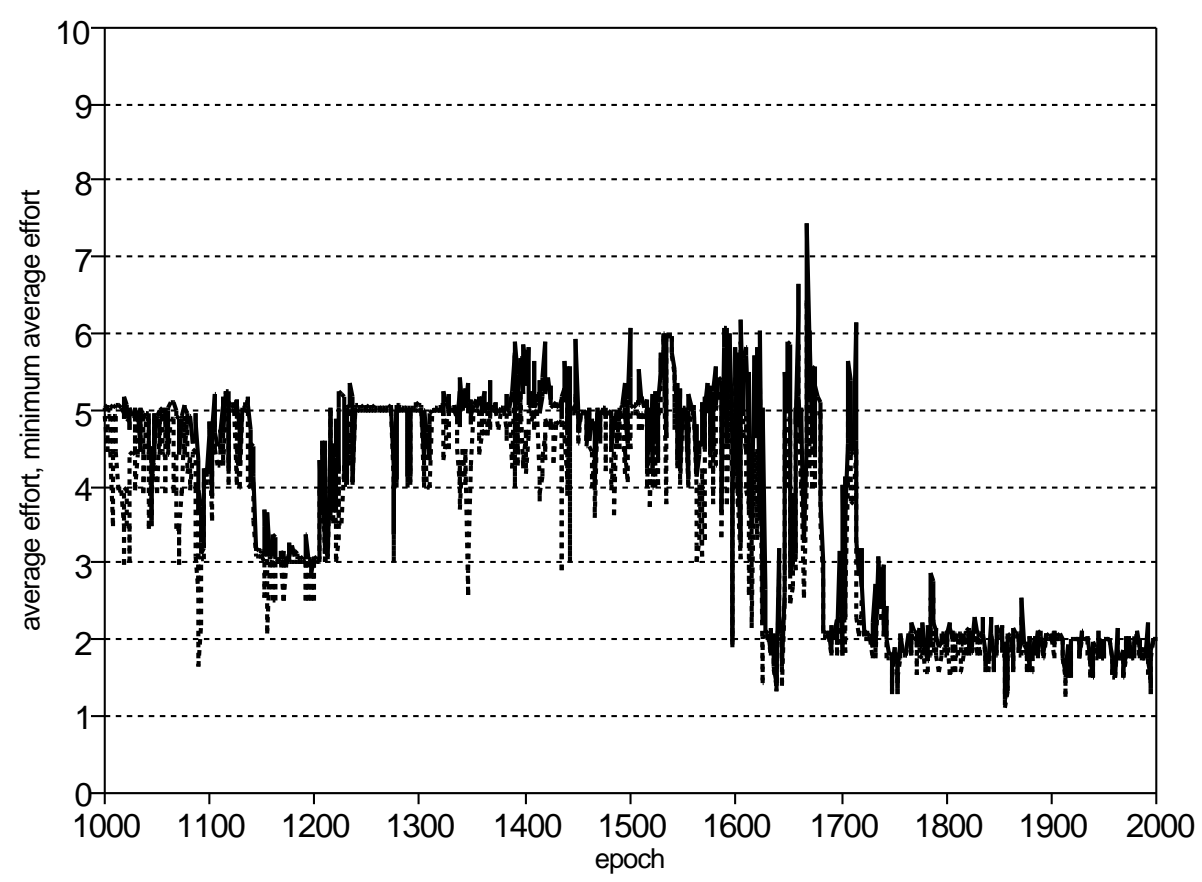

FiguRE 3. Average and average minimum effort; group size $=6$.

described feature of high frequencies of best response play was not preserved. Simulations with the probability of crossover set to zero (and $p_{m u t}=0.0033$ ) were characterized by lower frequencies of high levels of effort for all group sizes ${ }^{9}$.

The increase in the ratio of the payoff parameters $a$ and $b$ resulted in higher frequencies of high levels of effort for all group sizes. For example, setting $a=4$ and $b=1$, with $p_{\text {cross }}=0.6$ and $p_{\text {mut }}=0.0033$ resulted in $95.6 \%$ of the values of average effort, $\bar{e}_{h}$ between 7 and 8 , and $95.1 \%$ of the values of average minimum effort $\bar{e}_{\min , h}$ between 6 and 7 for the large group treatment, i.e. $g=14^{10}$.

Finally, the main features of the underlying dynamics should remain similar in case that different coding of players' strategies were implemented, i.e. if instead of binary number representation, integer numbers or real numbers were used to represent positions in players' strategies.

\footnotetext{
${ }^{9}$ The role of crossover has been extensively studied in the applications of the genetic algorithm to stationary optimization problems. However, its exact impact in economic applications where agents' actions affect the outcomes and outcomes feed back into actions is not well understood. This question requires comparative studies of the dynamics with and without crossover across a number of different economic environments.

${ }^{10}$ Complete sets of results for the above mentioned changes in the payoff parameters and parameters of the genetic algorithm are available from the author upon request.
} 


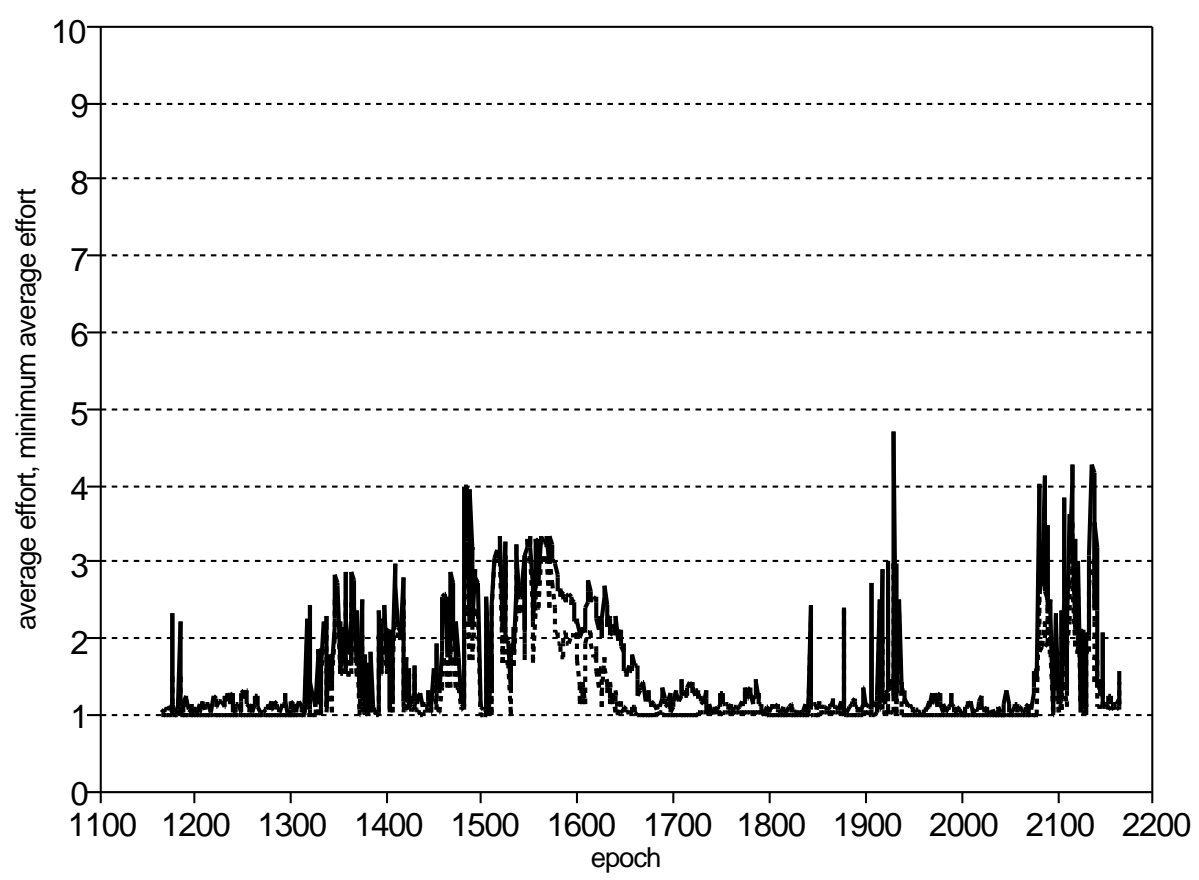

Figure 4. Average and average minimum effort; group size=6.

\section{Relevance of the MODEL FOR MACROECONOMiC ENVIRONMENTS}

It is worthwhile to point out two features of the dynamics generated by our model that are relevant for macroeconomic modeling.

First, as mentioned in the introduction, the team production game studied in this paper can be interpreted as a version of a model with strategic complementarities. This class of models is also characterized by multiplicity of equilibria that reflect different levels of economic activity and where the lack of coordination in agents' actions can result in bad equilibrium outcomes, i.e. economic recessions or even depressions. However, modeling persistent fluctuations in economic activity that result from changes in agents' expectations has proven to be quite difficult. A class of models that address this issue are the models with sunspot equilibria where shifts in agents' expectations are generated by an exogenous stochastic process. Thus, these models do not describe the transitions between periods of low and high economic activity and the process which leads to agents' coordination on a particular equilibrium.

Our evolutionary model results in the dynamics characterized by persistent economic fluctuations generated through changes in agents' expectations (without any changes in the fundamentals). Agents coordinate on good outcomes in 
TABle 4

\begin{tabular}{cccccccccc}
\hline$i$ & $p_{0}$ & $p_{1}$ & $p_{2}$ & $p_{3}$ & $p_{4}$ & $p_{5}$ & $p_{6}$ & $p_{7}$ & $p_{8}$ \\
\hline 1 & 5 & 5 & 3 & 1 & 5 & 5 & 3 & 1 & 1 \\
2 & 5 & 5 & 3 & 1 & 5 & 5 & 3 & 1 & 1 \\
3 & 5 & 5 & 3 & 1 & 5 & 5 & 3 & 1 & 1 \\
4 & 5 & 5 & 3 & 1 & 5 & 5 & 3 & 2 & 1 \\
5 & 5 & 5 & 3 & 3 & 6 & 5 & 3 & 1 & 1 \\
6 & 5 & 5 & 3 & 1 & 5 & 5 & 3 & 2 & 1 \\
7 & 5 & 5 & 3 & 1 & 5 & 5 & 3 & 1 & 1 \\
8 & 5 & 5 & 3 & 1 & 5 & 5 & 3 & 1 & 1 \\
9 & 5 & 5 & 3 & 1 & 5 & 5 & 3 & 5 & 1 \\
10 & 5 & 5 & 3 & 1 & 5 & 5 & 3 & 1 & 1 \\
11 & 5 & 5 & 4 & 1 & 5 & 5 & 3 & 1 & 1 \\
12 & 5 & 5 & 3 & 1 & 7 & 5 & 7 & 2 & 1 \\
13 & 5 & 5 & 3 & 1 & 5 & 5 & 3 & 1 & 1 \\
14 & 5 & 5 & 3 & 1 & 5 & 5 & 3 & 1 & 1 \\
15 & 5 & 5 & 3 & 1 & 5 & 5 & 3 & 1 & 1 \\
16 & 5 & 5 & 3 & 1 & 5 & 5 & 3 & 1 & 1 \\
17 & 5 & 5 & 3 & 1 & 5 & 5 & 3 & 2 & 1 \\
18 & 5 & 5 & 3 & 1 & 5 & 5 & 3 & 1 & 1 \\
19 & 5 & 5 & 4 & 1 & 5 & 5 & 3 & 1 & 1 \\
20 & 5 & 5 & 3 & 1 & 5 & 5 & 3 & 5 & 1 \\
21 & 5 & 6 & 3 & 1 & 5 & 5 & 3 & 2 & 1 \\
22 & 5 & 5 & 3 & 1 & 5 & 5 & 3 & 2 & 1 \\
23 & 5 & 5 & 3 & 1 & 5 & 5 & 3 & 2 & 1 \\
24 & 5 & 5 & 4 & 1 & 5 & 5 & 3 & 1 & 1 \\
25 & 5 & 5 & 3 & 1 & 5 & 5 & 3 & 1 & 1 \\
26 & 5 & 5 & 4 & 1 & 5 & 5 & 3 & 1 & 1 \\
27 & 5 & 5 & 3 & 1 & 5 & 5 & 3 & 1 & 1 \\
28 & 5 & 5 & 3 & 1 & 5 & 5 & 3 & 1 & 1 \\
29 & 5 & 5 & 3 & 1 & 5 & 5 & 3 & 1 & 1 \\
30 & 5 & 5 & 3 & 2 & 7 & 5 & 7 & 2 & 1 \\
\hline & & & & & & & &
\end{tabular}

some periods and fail to do so in other periods. In addition, from time to time, their strategies evolve towards one of the cyclical equilibria of the game. Thus, evolutionary behavior reported in this paper provides a framework for describing changes in agents' expectations and coordination on different equilibria.

Secondly, a possibility that populations of agents move between stationary and cyclical equilibria is an interesting one since, generally, in the economic models in which cycles exist, the convergence to cycles or steady states is determined by the type of the adaptive scheme that agents use. In the class of models of econometric learning, convergence to a cycle of order $k$ can be achieved if agents use separate averages for $k$ different phases of the cycle (for more detailed description 
TABle 5

\begin{tabular}{cccccccccc}
\hline$i$ & $p_{0}$ & $p_{1}$ & $p_{2}$ & $p_{3}$ & $p_{4}$ & $p_{5}$ & $p_{6}$ & $p_{7}$ & $p_{8}$ \\
\hline 1 & 1 & 7 & 1 & 8 & 6 & 1 & 2 & 3 & 3 \\
2 & 1 & 7 & 1 & 8 & 6 & 5 & 2 & 3 & 3 \\
3 & 2 & 7 & 1 & 8 & 2 & 5 & 4 & 3 & 3 \\
4 & 1 & 7 & 1 & 8 & 6 & 5 & 2 & 3 & 3 \\
5 & 1 & 7 & 1 & 8 & 6 & 1 & 2 & 3 & 3 \\
6 & 1 & 7 & 1 & 8 & 6 & 1 & 2 & 3 & 3 \\
7 & 1 & 7 & 1 & 8 & 6 & 1 & 2 & 3 & 3 \\
8 & 1 & 7 & 1 & 8 & 6 & 1 & 2 & 3 & 3 \\
9 & 2 & 7 & 1 & 8 & 2 & 5 & 4 & 3 & 3 \\
10 & 1 & 7 & 1 & 8 & 6 & 5 & 2 & 3 & 37 \\
11 & 2 & 7 & 1 & 8 & 2 & 5 & 4 & 4 & 3 \\
12 & 1 & 7 & 1 & 8 & 6 & 1 & 2 & 3 & 3 \\
13 & 2 & 8 & 1 & 8 & 2 & 5 & 3 & 3 & 3 \\
14 & 6 & 8 & 1 & 8 & 2 & 5 & 8 & 3 & 3 \\
15 & 1 & 7 & 1 & 8 & 6 & 5 & 2 & 4 & 3 \\
16 & 2 & 8 & 1 & 8 & 4 & 5 & 4 & 3 & 3 \\
17 & 1 & 7 & 1 & 8 & 6 & 1 & 2 & 3 & 3 \\
18 & 1 & 7 & 1 & 8 & 6 & 7 & 2 & 3 & 3 \\
19 & 2 & 7 & 1 & 8 & 2 & 5 & 4 & 3 & 3 \\
20 & 2 & 7 & 1 & 8 & 2 & 5 & 3 & 3 & 3 \\
21 & 2 & 8 & 1 & 8 & 2 & 5 & 8 & 3 & 3 \\
22 & 2 & 8 & 1 & 8 & 4 & 5 & 4 & 3 & 3 \\
23 & 2 & 8 & 1 & 8 & 2 & 5 & 4 & 3 & 3 \\
24 & 1 & 7 & 1 & 8 & 6 & 5 & 1 & 4 & 3 \\
25 & 2 & 7 & 1 & 8 & 2 & 5 & 4 & 3 & 3 \\
26 & 2 & 8 & 1 & 8 & 2 & 5 & 4 & 3 & 3 \\
27 & 1 & 7 & 1 & 8 & 6 & 1 & 2 & 3 & 3 \\
28 & 1 & 7 & 1 & 8 & 6 & 1 & 2 & 3 & 3 \\
29 & 2 & 7 & 1 & 8 & 2 & 5 & 3 & 3 & 3 \\
30 & 1 & 7 & 1 & 8 & 6 & 5 & 2 & 3 & 3 \\
\hline & & & & & & & &
\end{tabular}

of these algorithms and the results see Guesnerie and Woodford, 1991, and Evans and Honkapohja, 2001). Thus, agents can either learn a steady state of the model if they keep just one average that includes all past observations, or a cycle of order $k$ with $k$ separate averages. However, these models do not allow for the possibility that agents move between different types of equilibria while using the same adaptive technique. On the other hand, within our framework, the representation of agents' strategies and evolutionary adaptation allow transitions across stationary equilibria and cycles of different orders. 


\section{Concluding Remarks}

We formulate the minimum effort game as a repeated game and analyze the resulting equilibria. Not surprisingly, the set of equilibria becomes much larger then the set of equilibria of the stage game. There are both stationary and cyclical Nash equilibria. In addition, most of the Nash equilibria are weak. In terms of evolutionary stability, most of these equilibria are neutrally stable for monomorphic populations with a continuum of players and random, pairwise matching, implying that not all of the invading strategies will be driven out of the population.

The analysis of the long-run behavior of the economies which adapt under the genetic algorithm shows that, regardless of the group size, any equilibrium can be reached. Due to the larger amount of strategic uncertainty, economies with large-size groups spend most of the time in the low effort equilibria. Economies in which players play in small groups that are characterized by a smaller amount of strategic uncertainty spend more time close to the high effort equilibria. At the same time, regardless of the group size, all populations spend significant amount of time playing best response actions which implies that they spend most of the time close to one of the Nash equilibria of the repeated game.

The models based on evolutionary game theory and adaptive behavior demonstrated the convergence to the equilibrium with the minimum level of effort in the team production game. However, they could not account for the payoff dominant equilibrium outcome of the experiments with small group treatments as well as for the experimental evidence that large groups can reach higher effort equilibria.

The model presented in this paper is capable of capturing the main features of the behavior observed in the experiments with human subjects: convergence to the minimum level of effort for large group treatments, convergence to the maximum level of effort for fixed-pairs treatments, and a possibility that large groups reach higher effort equilibria if the game is played long enough.

Even though the model described in the paper is not a fully blown macroeconomic model, its dynamics with persistent fluctuations in the level of effort suggest the framework within which we can actually generate persistent fluctuations in the level of macroeconomic activity that are driven by animal spirits, i.e. changes in agents' expectations that are unrelated to economic fundamentals.

Acknowledgements. I gratefully acknowledge financial support from the Social Sciences and Humanities Research Council of Canada.

\section{REFERENCES}

Arifovic J. (1994) Genetic Algorithm Learning and the Cobweb Model. J. Econ. Dynamics and Control 18, 3-28.

Arifovic J. (1996a) The Behavior of the Exchange Rate in the Genetic Algorithm and Experimental Economies. J. Political Economy 104, 510-541. 
Arifovic J. (1996b) Strategic Uncertainty and the Genetic Algorithm Adaptation, in Computational Approaches to Economic Problems, eds. by H. Amman, B. Rustem and A. Whinston, Kluwer Academic Publishers, p. 225-237.

Arifovic J. and Ledyard J. (2000) Evolutionary Learning and Mechanism Design, manuscript.

Back Th. (1996) Evolutionary algorithms in theory and practice: evolution of strategies, evolutionary programming, genetic algorithms. New York: Oxford University Press.

Berninghaus S. and Ehrhart K. (1998) Time horizon and equilibrium selection in tacit coordination games: Experimental results. J. Econ. Behavior and Organization 37, 231-248.

Bryant J. (1983) A Simple Rational Expectations Keynes-Type Model, Quart. J. Econom. 98, 525-529.

Cooper R. and John A. (1988) Coordinating Coordination Failures in Keynesian Models, Quart. J. Econom. 103, 441-463.

Cooper R. (1999) Coordination Games, Cambridge, England: Cambridge University Press.

Crawford V. (1991) An 'Evolutionary' Interpretation of Van Huyck, Battalio, and Beil's Experimental Results on Coordination, Games and Economic Behavior 3, 25-59.

Crawford V. (1995) Adaptive Dynamics in Coordination Games. Econometrica 63, 103-143.

Eaton C. and Morrison W. (1998) On Evolution, Subgame Perfection and Retaliation, manuscript.

Evans G. and Honkapohja S. (2001) Learning and Expectations in Macroeconomics, Princeton: Princeton University Press.

Guesnerie R. and Woodford M. (1991) Stability of Cycles with Adaptive Learning Rules, In Equilibrium Theory and Applications, Proceedings of the Sixth International Symposium in Economic Theory and Econometrics, W. Barnett et al., eds., Cambridge University Press, Cambridge, 111-134.

Holland J.H. (1975) Adaptation in Natural and Artificial Systems. Ann Arbor: The University of Michigan Press.

Kandori M., Mailath G. and Rob R. (1993) Learning, Mutation and Long Run Equilibria in Games. Econometrica 61, 29-56.

Roth A. (1995) Introduction to Experimental Economics, 3-109 in Kagel J. and Roth A. (eds.), Handbook of Experimental Economics. Princeton University Press.

Van Huyck J., Battalio R. and Beil R. (1990) Tacit coordination games, strategic uncertainty, and coordination failure. Amer. Econom. Rev. 80, 234-248.

Weibull J. (1995) Evolutionary Game Theory. Cambridge, MA: MIT Press.

Young H.P. (1993) The Evolution of Conventions. Econometrica 61, 57-84. 